\title{
INTRODUCCIÓN A LA TECNOLOGÍA DE FIBRAS ÓPTICAS Y ANÁLISIS NUMÉRICO DE LA PROPAGACIÓN DE LA L.UZ EN FIBRAS MICRO-ESTRUCTURADAS
}

\author{
Víctor H. ARISTIZÁBAL ${ }^{1}$
}

\section{Resumen}

En este trabajo se presenta una amplia introducción acerca de las fibras ópticas, que por tratarse de una tecnología que aún está en fase de desarrollo, es importante realizar estudios teóricos para predecir la respuesta óptica y diseñar nuevos sistemas que solucionen problemas en telecomunicaciones y sensórica. Para este fin, se emplea una herramienta de cómputo basada en el método escalar de elementos finitos para analizar cualquier tipo de guías de onda dieléctricas donde, particularmente, se estudian las fibras ópticas micro-estructuradas, fibras muy especiales porque consisten en un medio homogéneo, con un arreglo de huecos de aire que se mantiene a lo largo de su estructura, permitiendo, en general, la utilización de métodos numéricos vectoriales para su análisis. Luego, se enuncia una breve introducción a la metodología utilizada, para los análisis numéricos. Finalmente, para una fibra óptica micro-estructurada con huecos de forma anular, se comparan los resultados obtenidos con los reportados en la literatura.

1 Ingeniero Físico y Magíster en Ciencias Físicas, Universidad Nacional de Colombia, sede Medellín. Doctorando en Ingeniería, Universidad EAFIT - sede Medellín.

E-mail: vharisti@yahoo.com 


\section{Palabras clave}

Modelación numérica de fibras ópticas, Método de elementos finitos, Fibras ópticas, Fibras de cristal fotónico, Fibras microestructuradas, Dispersión.

\section{Abstract}

This written shows a huge induction about the optical fibers, which for being a technology under development, is important to study theorically to predict the optical answer and design new systems which solve the Telecommunications and sensorial. That's why we use a computer tool based in finite elements scaling method to analyze any kind of dielectric waves guides where particularly the micro-structured optical fibers are studied, very special fibers because they consist on an homogeneous ways with air holes arrangement that exit all over their structure, helping generally to the use of vectorial numeric methods for their analysis. Then, we enunciate a brief introduction the methodology used for that numerical analysis. Finally, for a micro-structured optical fiber with annular form holes, the results are compared with the reported in the literature.

\section{Key words}

Optical fibers numeric modeling, Finite elements methods, Optical fibers, Photonic crystal fibers, Micro-structured fibers, Dispersion. 


\section{INTRODUCCIÓN}

Durante las últimas décadas, los avances en el campo de las telecomunicaciones y la sensórica han venido de la mano con la implementación de una tecnología revolucionaria, que ha alcanzado difusión a escala mundial y cuyo desarrollo aún no se ha detenido en virtud al interés que ha despertado en el ámbito científico. Lógicamente se habla de los dispositivos ópticos, los cuales son utilizados para procesos, monitoreo y control de sistemas (Derickson: 1998), y de las fibras ópticas; aquel medio tan grueso como un cabello que guía la luz a través de enormes distancias, transportando datos de manera tan eficiente y con las ventajas que, frente a otras tecnologías, la ubicaron en la importante posición actual.

Los sistemas de fibra óptica utilizan la energía óptica como soporte, por lo que presentan un conjunto importante de ventajas sobre otros soportes utilizados en la transmisión y tratado de señales analógicas y digitales. Entre ellas están:

- Alta sensibilidad a los cambios que se producen en su entorno

- Gran ancho de banda, que permite la transmisión de un gran volumen de información a altas velocidades

- Atenuación baja. Las pérdidas en intensidad son menores, lográndose mayor alcance

- Inmunidad a interferencias electromagnéticas. La fibra óptica es absolutamente inmune a las interferencias de radiofrecuencias e impulsos electromagnéticos, lo que minimiza errores en la transmisión de señales digitales. Esto es de gran importancia en aplicaciones de control industrial donde se genera gran cantidad de ruido

- Seguridad y aislamiento eléctrico. En determinadas aplicaciones para ambientes peligrosos (ambientes explosivos o inflamables) o en electromedicina, las fibras ópticas son imprescindibles debido a la imposibilidad de producir descargas eléctricas o chispas 
- Menor peso y volumen. Comparando las fibras ópticas y los cables coaxiales necesarios para obtener las mismas prestaciones, las primeras ocupan un volumen muy inferior y tienen menor peso

- Seguridad frente a posibles intervenciones de la línea. Aunque no es imposible intervenir una fibra óptica, resulta más difícil que en otros medios y es fácilmente detectable

El actual abanico de aplicaciones de las fibras ópticas es muy amplio y continúa en aumento; no obstante, dos de los campos de mayor importancia son:

1. Telecomunicaciones: Por su gran ancho de banda, la fibra óptica es el medio de transmisión preferido: en la actualidad las fibras comunican continentes y regiones apartadas a través de cables que se han esparcido por océanos, aire y tierra para unir a todo el mundo en la comunidad global que hoy tenemos. Esto, a su vez, ha impulsado el desarrollo de nuevos dispositivos relacionados con esta tecnología como circuladores ópticos, amplificadores, compensadores, multiplexores, demultiplexores, WDM, aisladores, controladores de polarización, fuentes de luz, etc. (Schott, 2008).

2. Sensores: Se han desarrollado dispositivos capaces de medir parámetros físicos como presión, temperatura, deformaciones mecánicas, entre otros. Que son utilizados en los sectores eléctrico, gas, petróleo, civil, medicina, aeroespacial, automotriz, químico, metrología y control, medio ambiente, etcétera. (GAVEA SENSORS, 2008).

\subsection{Fibras ópticas convencionales}

Las fibras ópticas convencionales corresponden a aquella estructura de silice con un grosor cercano al de un cabello humano, con un núcleo como región primaria de guiado de la luz, cuyo diámetro alcanza unas cuantas micras. En el núcleo la composición 
del vidrio se altera por medio de un dopaje, de manera que el valor del índice de refracción es un poco mayor al del revestimiento, es decir, el medio que lo circunda (Ver Figura 1). Esta diferencia de índice es la que permite que se dé el fenómeno de la reflexión total interna, mediante el cual la luz que se propaga por la fibra se mantiene mayoritariamente confinada en su núcleo.

Figura 1. Estructura de UnA Fibra ÓPtica CONVENCIONAL

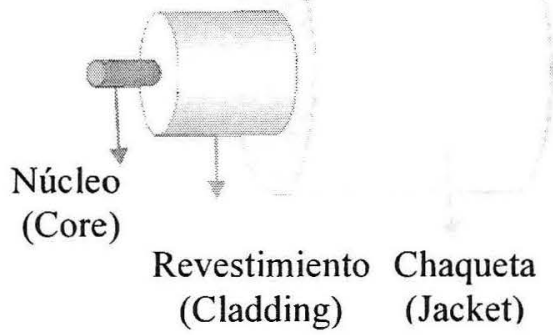

Debido a la naturaleza ondulatoria de la luz, con la propagación se manifiesta un efecto característico de resonancia. Este caso se evidencia con la aparición de ciertas distribuciones de campo óptico preferentes (Figura 2) a las que se denominan modos de propagación y los cuales son determinados resolviendo las ecuaciones de Maxwell. Visto desde la teoría de rayos, las diferentes trayectorias que puede seguir un haz de luz en el interior del núcleo de la fibra se denominan modos de propagación (Figura 3). El primer modo, o "modo fundamental", con una distribución del campo electromagnético cuasi-gaussiano, es el único soportado en fibras ópticas estándar para telecomunicaciones a la longitud de onda del infrarrojo comúnmente usada, $1550 \mathrm{~nm}$.

Hay diferentes tipos de fibras "convencionales". La estructura más simple es la de perfil de índice de refracción de paso, o escalonado, cuyo valor cambia abruptamente en la interfase núcleo-revestimiento. Esta a su vez se divide en los dos tipos más empleados, denominados en virtud de sus características de 
Figura 2. Distribuciones EN INTENSIDAD dEL CAMPO ÓPTICO DE ALGUNOS MOdOS DE PROPAGACIÓN EN UNA FIBRA DE PERFIL DE INDICE DE PASO, CALCULADOS CON LA METODOLOGIA PROPUESTA EN ESTE TRABAJO

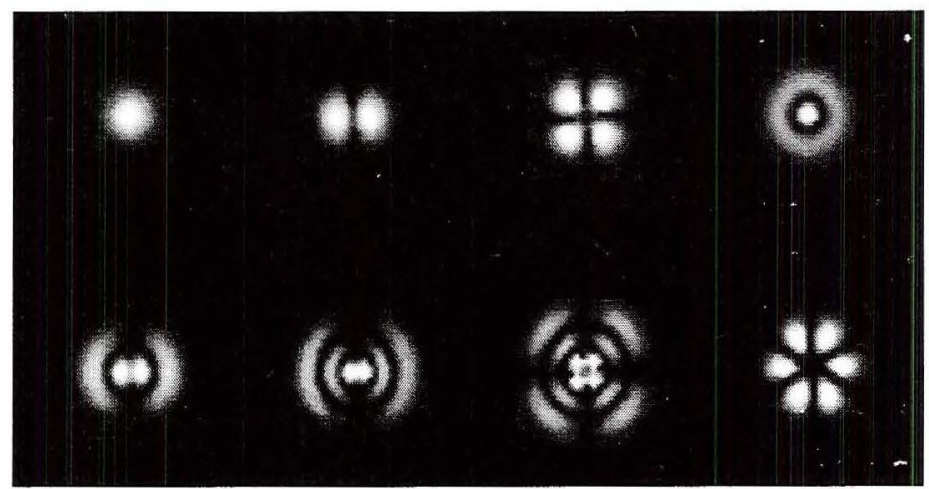

Figura 3. REPRESENTACIÓN DESDE EL PUNTO DE VISTA DE LA TEORIA DE RAYOS DE LA PROPAGACIÓN CARACTERISTICA DE LA LUZ AL INTERIOR DEL NÜCLEO DE UNA FIBRA ÓPTICA. ESQUEMA TOMADO DE (NETWORK CABLING HELP, 2008)

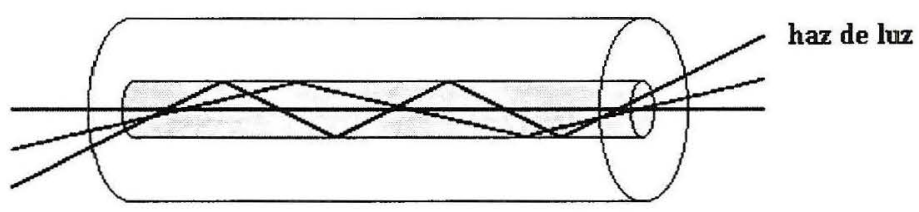

transmisión: mono-modo, ver Figura 4(c), y multi-modo, ver Figura 4(a), que, desde un punto de vista práctico, se diferencian en el diámetro de su núcleo, que en el caso de la primera se encuentra, por lo general, por debajo de las 10 micras, mientras que en el de las segundas, suele estar por encima de las 50 micras.

Hay otra estructura un poco más compleja que la anterior, la denominada fibra de índice gradual: aquí el índice de refracción tiene un valor máximo en el eje de la fibra y va disminuyendo suavemente hasta el revestimiento. La consecuencia de este tipo de perfil es que cualquier rayo de luz que se propague por la fibra, según se aprecia en la Figura 4(b), va sufriendo una deflexión 
progresiva en su camino, de tal forma que la trayectoria que sigue tiene una forma ondulatoria.

En telecomunicaciones, el comportamiento multi-modal es indeseado porque cada modo viaja a diferente velocidad a lo largo de la fibra, ocasionando que un pulso de luz bien definido que ingrese al guía de onda, tras propagarse por una distancia considerable, resulte dispersado a causa del retraso relativo entre los diversos modos que lo componen. A este efecto se le conoce como dispersión modal. Más aún, si se considera el caso en el que solamente se propaga el modo fundamental, se encuentra con una circunstancia similar, puesto que el rango de longitudes de onda del que espectralmente está compuesto un pulso de luz, da origen a la dispersión cromática en razón al retraso relativo que experimentan las diferentes ondas que lo integran.

Figura 4. Tipos de fibras ópticas CONVENCIONALES.

ESQUEMA TOMADO DE LA REFERENCIA (YIO: 2008)

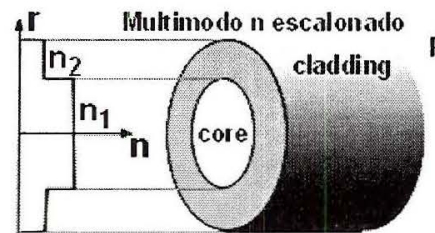

ir Multimodo n gradual

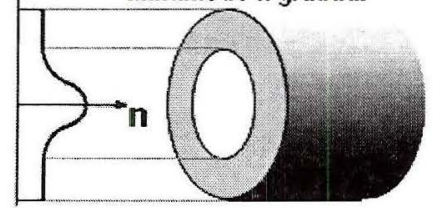

4 Mlonomodo $n$ escalonado

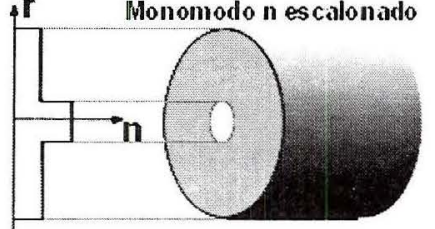

Pulso de entrada
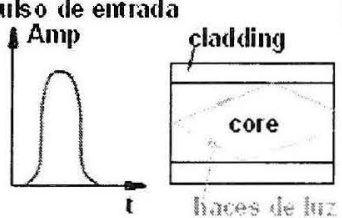

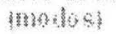
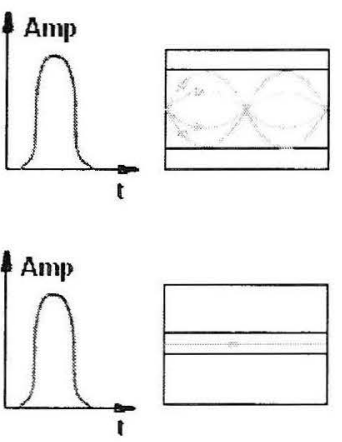

Pulso de sadida

Amp

(a)

Amp

(b)

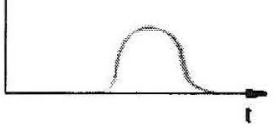

(c)

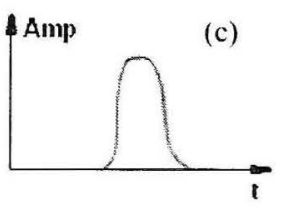


Este comportamiento de la dispersión, así como muchas otras características de las fibras convencionales, ha venido siendo replanteado con el desarrollo de las fibras micro-estructuradas, con las cuales se han obtenido importantes avances, tanto en el terreno de las telecomunicaciones como en otros campos.

\subsection{Fibras ópticas micro-estructuradas}

La tecnología de las fibras ópticas ha avanzado rápidamente en los últimos años; mientras los principios básicos del guiado de la luz mediante reflexión total interna han sido conocidos desde hace mucho tiempo, la capacidad para fabricar hilos muy pequeños con estructuras complejas de silicio de alta pureza ha sido recientemente alcanzada. Paralelo a este desarrollo en la manufactura de guías de onda, actualmente, los campos de la investigación y de la ingeniería están concentrados en el diseño y optimización de nuevas guías de onda estándar con micro-estructuras que provean soluciones a aplicaciones prácticas en telecomunicaciones, tales como el control de la dispersión, disminución de las pérdidas y la operación en el régimen mono-modo; y en sensórica el de proveer estructuras sensibles a parámetros de interés y que sean de fácil control. Todo esto ha sido propiciado por ideas novedosas, acompañadas de un estudio que típicamente se ha llevado a cabo por medio de modelos computacionales.

Orientando toda la atención en las guías de onda ópticas micro-estructuradas, especialmente en el grupo de las fibras (estructuras con sección transversal circular), se encuentran las Fibras de Cristal Fotónico (Photonic Crystal Fibers o PCFs) (Russell et al, 2003), las cuales son hechas de silice puro y tiene una región central rodeada por múltiples huecos de aire, dispuestos de manera periódica que corren a lo largo de la longitud de la fibra. Las PCFs pueden dividirse en dos clases de fibras. En la primera, la región central es sólida y haría de núcleo y la región que tiene múltiples huecos de aire dispuestos de forma periódica haría de revestimiento (Knight et al, 1996, 1997), tal como se muestra en la Figura 5(a), donde el guiado de la luz se da mediante reflexión total interna a 
través del núcleo sólido, siendo mayor el índice de refracción en el núcleo que en el revestimiento. Del otro lado, en la Figura 5(b) se muestra la segunda clase de PCF, donde la región central es de aire y en la de revestimiento los huecos de aire también están dispuestos de forma perfectamente periódica (Knight et al, 1998, Cregan et al, 1999), pero en estas estructuras se presenta el efecto denominado Brecha de Banda Fotónica ${ }^{2}$ (Photonic Band Gap) a la longitud de onda de operación de la fibra, haciendo posible el guiando de la luz por el núcleo de aire, el cual tendría un índice de refracción menor que el revestimiento. En este mecanismo, se transmiten selectivamente, ciertos anchos de banda espectrales, es decir, ciertos rangos de longitud de onda, de tal manera que si se inyecta luz blanca a la fibra, a su salida se obtendrá luz con determinados colores.

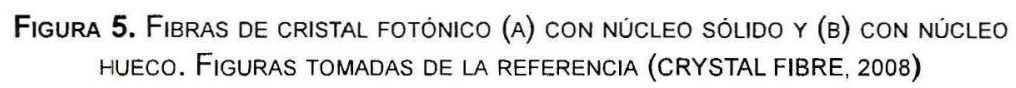

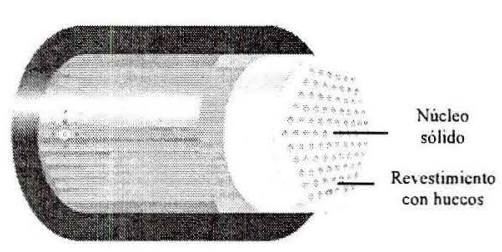

(a)

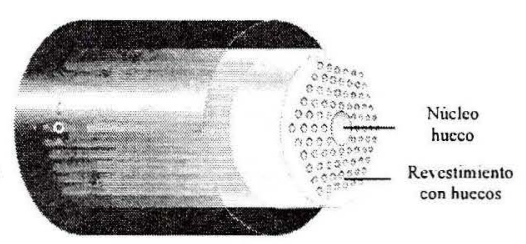

(b)

Desde la introducción de las fibras de cristal fotónico, se han realizado diferentes estructuras que guían señales ópticas utilizando arreglos de huecos micro-estructurados. Algunos ejemplos de fibras con huecos se muestran en la Figura 6 (Holey Fibers o

\footnotetext{
Los arreglos periódicos de huecos presentes en los cristales fotónicos afectan la propagación de las ondas electromagnéticas de la misma manera como el potencial periódico, en un cristal semiconductor, afecta el movimiento de electrones definiendo bandas de energía electrónicas permitidas y prohibidas.
} 
HFs es una etiqueta más amplia para estos tipos de fibras debido a que los arreglos de huecos no tienen que ser necesariamente periódicos). En estas estructuras, el índice de refracción efectivo de los modos propagantes es un parámetro esencial, pues determina, entre otras, las propiedades de dispersión de la guía de onda. Actualmente, las fibras con huecos están atrayendo la atención debido a que presentan propiedades únicas, que no son realizables con las fibras ópticas convencionales.

Figura 6. Fibras con huecos (HOLEy Fibers o HF). Figuras tomadas de las REFERENCIAS (LASER FOCUS WORLD: 2008, PRECISION TECHNOLOGIES, 2008, THOMASNET, CRYSTAL FIBRE, 2008)

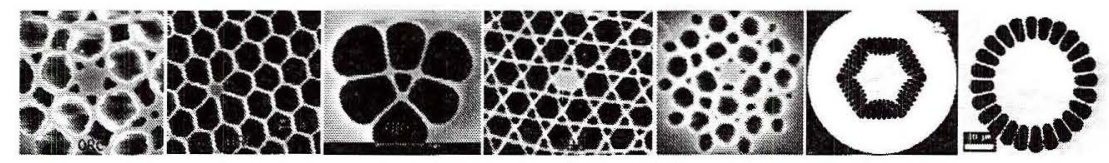

La primera HF fue propuesta en 1992 y solamente hasta 1995 se hizo posible fabricarlas; en 1996 se reportó el primer trabajo con estas fibras especiales (Knight et al, 1997). El procedimiento de fabricación (ver Figura 7) se basa en el apilamiento de tubos y barras de silicio en una estructura macroscópica denominada preforma, con el patrón de agujeros requeridos en la fibra definitiva. La preforma es luego compactada e introducida en el horno de una torre de estiramiento, alcanzando una temperatura de ablandamiento cercana a los $2000^{\circ} \mathrm{C}$. En este punto, la preforma es fundida y estirada mediante un carrete, adquiriendo un diámetro de $1 \mathrm{~mm}$ con agujeros de alrededor $0.05 \mathrm{~mm}$. Hasta aquí, la estructura es una versión reducida en un factor de 20 de la preforma que llega a alcanzar las dimensiones definitivas tras experimentar un segundo estiramiento, haciendo que el espaciamiento entre agujeros puede ser de una micra, con agujeros de diámetro de 0.25 micras, y así para fibras de varios kilómetros de longitud. El proceso descrito brinda la posibilidad de jugar ampliamente con la geometría y 
distribución de los agujeros en la fibra, lo que a su vez hace posible la manipulación de las propiedades ópticas de los modos guiados. Finalmente, la fibra es recubierta con una chaqueta estándar que provee una manipulación segura de la fibra, para que no se estropee.

Figura 7. Fabricación de las HF. Esquema tomado de La ReFerencia (CRYSTAL FIBRE: 2008)

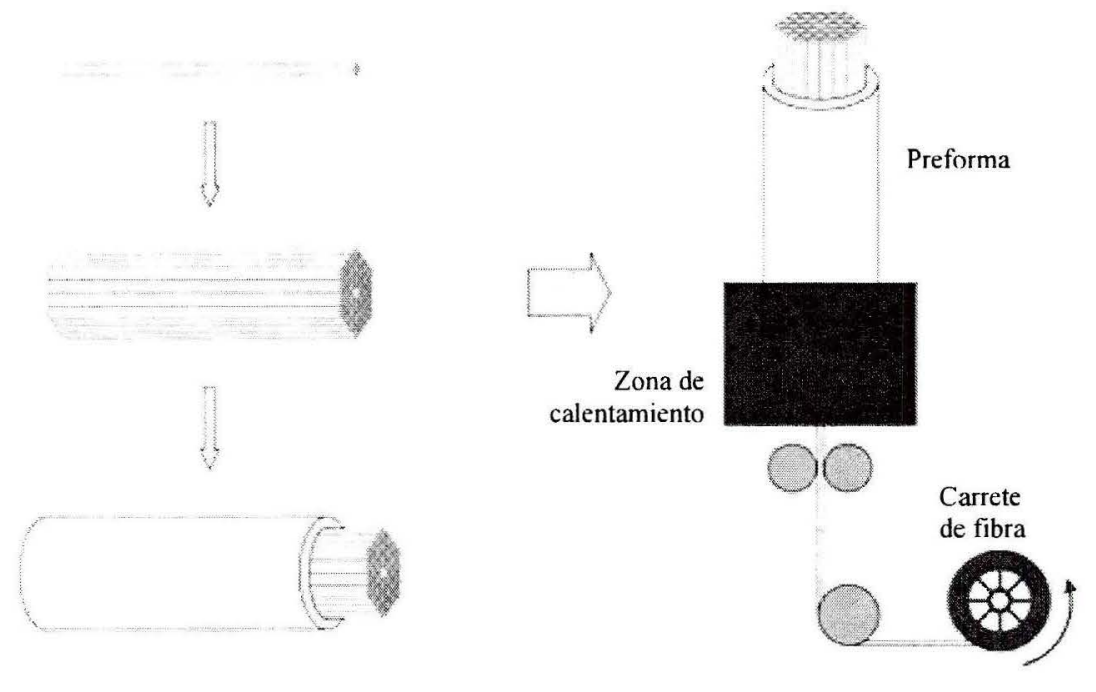

Para tener una visión más amplia de la importancia de estos tipos de guías de onda, se mostrarán aplicaciones específicas con estas fibras. Algunas, han sido reportadas por los líderes tecnológicos como Corning, Bell Labs (Lucent Technologies), OmniGuide Communications (EE.UU.), Crystal Fibre (Dinamarca) o BlazePhotonics (Gran Bretaña). Adicional a esto, y al margen de sus alcances en el terreno de las telecomunicaciones, las guías de onda micro-estructuradas han abierto la puerta a múltiples e interesantes aplicaciones, algunas de ellas son: 
- Manejo de la dispersión. La estructura de las fibras con huecos ha permitido manipular la dispersión en función de la longitud de onda, al punto de lograr dispersión nula para longitudes dentro del rango del visible, valores muy altos de dispersión anómala cerca al infrarrojo (Gander et al, 1999; Knight et al, 2000; Huttunen \& Torma, 2005) y dispersión cromática ultraplana en grandes intervalos de longitud de onda (Reeves et al, 2005; Saitoh et al, 2003; Penn Well Corporation, 2004), cosa que no ocurre con una fibra convencional.

- Operación mono-modo. Se han diseñado estructuras donde se ha logrado que la fibra pueda operar en régimen mono-modo en rangos de longitud de onda muy amplios (Knight et al, 1996; Birks et al, 1997; Penn Well Corporation, 2004). Adicionalmente, se ha logrado que estas estructuras tengan una gran área modal y, de esta manera, lograr transmitir altas potencias de luz, sin que se presenten efectos no lineales y ningún peligro, debido a la baja densidad de potencia óptica.

- Control de polarización. Una disposición específica de los agujeros que rodean al núcleo hace que se induzca birrefringencia en los modos guiados (Penn Well Corporation, 2004; Ortigosa et al, 2004; Saitoh \& Koshiba, 2003), lo que ocasiona que la luz viaje a diferentes velocidades dependiendo de su estado de polarización. Los valores de birrefringencia aquí obtenidos superan en un orden de magnitud a los obtenidos con las fibras convencionales.

- Efectos no lineales. Con algunas de estas fibras es posible obtener efectos no lineales ${ }^{3}$ muy fuertes (Penn Well Corporation,

Algunos de estos efectos se producen debido a la dependencia del índice de refracción con la intensidad del campo aplicado, que se vuelven importantes cuando la potencia óptica es elevada: auto modulación de fase (SPM), modulación de fase cruzada (CPM) y la mezcla de cuarta onda (FWM). También, se generan por efectos de dispersión en la fibra, debido a la interacción de las ondas de luz con los fonones (vibraciones moleculares) en el silicio: dispersión Raman estimulada (SRS) y la dispersión Brillouin estimulada (SBS). 
2004; Fujisawa \& Koshiba, 2003), al punto de transformar un pulso corto invisible de luz infrarroja $(0.2 \mathrm{ps}, 50 \mathrm{~kW})$ en un "estallido" de luz con el espectro de la luz solar pero 10.000 veces más brillante. Aplicaciones al respecto se están desarrollando, entre otros, en espectroscopia e imagen diagnóstica.

- Fibras con bajas pérdidas. Las fibras con núcleo hueco (Knight et al, 1998; Penn Well Corporation, 2004; Argyros et al, 2004) tienen el potencial de eliminar, casi por completo, las no linealidades, responsables de perturbaciones en la transmisión de datos, así como de reducir drásticamente los niveles de pérdidas, ocasionadas, en gran medida, por el esparcimiento Rayleigh ${ }^{4}$, con lo cual se plantea un cambio fundamental en la estructura de las telecomunicaciones a gran escala, al disminuir los requerimientos de dispositivos tales como amplificadores y repetidores, reduciendo así los costos en equipamiento e instalación.

Como se puede ver, la importancia de analizar la propagación característica de la luz en estas guías especiales es incuestionable; se trata de una tecnología que aún está en etapa de desarrollo, por lo que un estudio teórico, bien sea analítico o numérico, es de vital importancia, ya que se podría predecir el comportamiento óptico de aquellas estructuras. Debido a la gran variedad de posibles formas y arreglos para los huecos, que dan lugar a estructuras bastante complejas que no tienen solución analítica, se demanda el uso de métodos numéricos que permitan modelar adecuadamente la sección transversal de estas fibras, para analizarlas y, así, diseñar nuevas estructuras para futuras aplicaciones.

Adicionalmente, la existencia de interfases con alto contraste en el índice de refracción entre el sílice (alrededor de 1.5) y los huecos de aire (1.0), hace necesario considerar una solución vectorial para tomar en cuenta los efectos de polarización del

\footnotetext{
1 Este fenómeno es debido a irregularidades a nivel molecular en la estructura del vidrio.
} 
campo óptico. Para este fin se han propuesto métodos vectoriales semi-analíticos (Silvestre et al, 1998; Mogilevtsev et al, 1999; Monro et al, 2000; Steel et al, 2001; Campbel et al, 2004; Argyros et al, 2004; Alivizatos et al, 2004) y métodos vectoriales numéricos (Yu et al, 2004; Brechet et al, 2000; Saitoh \& Koshiba, 2002; Cucinota et al, 2002; Rahman \& Davies, 1984; Koshiba \& Tsuji, 2000). Mientras que los métodos semi-analíticos intentan modelar tanto el campo electromagnético como la distribución del índice de refracción en estas estructuras mediante funciones analíticas (funciones bases), los métodos totalmente numéricos los modelan mediante técnicas especiales, para llegar a algoritmos bastantes complejos y exigentes, computacionalmente hablando. La ventaja principal de los métodos semi-analíticos es que permiten un análisis más liviano y menos exigente en recursos, que los numéricos; pero su aplicabilidad está limitada a un pequeño grupo de estructuras que presentan periodicidad y altas simetrías de los huecos, situación que no se presenta en la mayoría de los métodos numéricos.

También, se han reportado métodos escalares para el análisis de fibras micro-estructuradas (Monro et al, 1999; Kerbage et al, 2000; Riishede et al, 2003; Birks et al, 2004). No obstante, estas aproximaciones son incapaces de predecir propiedades ópticas tales como la birrefringencia, por lo tanto, empiezan a perder precisión para longitudes de onda comparables con la separación y tamaño de los huecos. Su atractivo se debe a que los requerimientos y procesos computacionales son mucho menores que en las técnicas vectoriales.

Como una alternativa más eficiente a las soluciones vectoriales, en este trabajo se propone (Aristizábal et al, 2004, 2006) considerar que el campo electromagnético está linealmente polarizado y contenido en la sección transversal de la guía de onda. Además, al no considerar los efectos de acoplamiento de las componentes del campo, se llega a una ecuación de onda escalar, la cual se soluciona con el Método de los Elementos Finitos (FEM), que es una técnica que permite modelar con mucha precisión cualquier estructura que tenga la guía de onda. Luego de realizar la solución 
escalar y adicionar una corrección, de primer orden, que incluye los efectos de polarización causados por la discontinuidad en el índice de refracción en las interfases sílice-aire presentes en estas estructuras, se mejora la precisión de los resultados, ampliando su rango de validez a longitudes de onda largas y fracciones de llenado de aire grandes en fibras micro-estructuradas. Evidentemente, esta aproximación es más eficiente que los métodos de cálculo completamente vectoriales, ya que toma ventaja de la simplicidad de la aproximación escalar. Para este propósito se usaron elementos triangulares de primer orden, cuya región que delimitan, es de índice de refracción homogéneo, y se aprovecha el hecho de que el FEM provee directamente la distribución del campo sobre la sección transversal de la fibra, para la corrección al análisis escalar.

En este trabajo se quiere mostrar la validez de la metodología numérica propuesta, analizando una fibra de cristal fotónico multimodo, estructura completamente diferente a las típicas analizadas con huecos circulares, como una manera de aproximarnos a analizar guías de onda ópticas micro-estrucutradas. Por considerarlo de interés, primero se presenta una descripción del método y luego se muestran los resultados obtenidos para el parámetro de dispersión de esta estructura reportada en la referencia (Light Reading, 2008).

\section{DESCRIPCIÓN DEL MÉTODO}

\subsection{Métorio de Elementos Finitos Escalar}

El campo eléctrico de un modo de propagación en una guía de onda arbitraria se puede expresar como

$$
\vec{E}(x, y, z)=\left\{\vec{E}_{\imath}+E_{z} \hat{z}\right\} \exp (i \beta z),
$$

donde $B$ es la constante de propagación, $\hat{z}$ es el vector unitario paralelo al eje de la guía, $\vec{E}_{t}=E_{x} \hat{x}+E_{y} \hat{y}$ y $E_{z}$ son las componentes transversal y longitudinal del campo eléctrico, respectivamente. Si trabajamos el campo como se describe en (1) en la ecuación de 
onda vectorial, fácilmente se puede demostrar que la componente transversal del campo eléctrico satisface la ecuación de onda

$$
\left\{\nabla_{t}^{2}+k_{0}^{2} n^{2}(x, y)-\beta^{2}\right]_{\mathbf{E}}=-\nabla,\left\{\overrightarrow{\mathbf{E}}_{t} \cdot \nabla_{t} \ln n^{2}\right\}
$$

donde $\nabla_{t}^{2}$ es el operador Laplaciano en el plano transverso, $n=n(x, y)$ es el perfil del índice refracción, y $k_{0}=2 \pi / \lambda$ el número de onda en el vacío, siendo $\lambda$ la longitud de onda.

La aproximación escalar consiste en despreciar los efectos de acoplamiento de las componentes del campo, haciéndose cero el término a la derecha de la ecuación (2), quedando

$$
\left\{\nabla_{t}^{2}+k_{0}^{2} n^{2}-\widetilde{\beta}^{2}\right\} \overrightarrow{\mathbb{E}}_{t}=0,
$$

donde $\vec{E}_{t}$ y $\widetilde{\beta}$ son el campo escalar y su correspondiente constante de propagación, respectivamente.

Para solucionar la ecuación (3) se usa el método de elementos finitos, que convierte la ecuación (3) en el problema matricial de valores propios generalizado:

$$
[A]\{e\}=\not^{2}[B]\{e\},
$$

donde el autovector $\{e\}$ contiene los valores del campo eléctrico en cada uno de los vértices de los elementos triangulares utilizado en la discretización de la sección transversal de la guía de onda. Las matrices $[\mathrm{A}]$ y $[\mathrm{B}]$ son de la forma

$$
\begin{gathered}
{[A]=\sum_{e} \iint_{e}\left(k_{0}^{2} n_{e}^{2}\{N\}^{\top}\{N\}-\frac{\partial\{N\}^{\mathrm{T}}}{\partial x} \frac{\partial\{N\}}{\partial x}-\frac{\partial\{N\}^{\mathrm{T}}}{\partial y} \frac{\partial\{N\}}{\partial y}\right) d x d y,} \\
{[B]=\sum_{e} \iint_{e}\{N\}^{\mathrm{T}}\{N\} d x d y .}
\end{gathered}
$$

La sumas en (6) y (7) corren sobre todos los elementos denotados por e. El índice de refracción $n(x, y)$ para cada elemento se considera uniforme e igual a $n_{e}$, $[\mathrm{N}]$ es el vector de funciones de forma. 


\subsection{Correccion por polarización}

La corrección por polarización a la constante de propagación proviene, como se concluye de la ecuación (2), de la discontinuidad del índice de refracción. Para esta corrección se define la relación entre la constante de propagación $\beta$ de la ecuación de onda vectorial (2) y $\widetilde{\beta}$ de la ecuación de onda escalar (3). Para modos polarizados en $\mathrm{x}$ o $\mathrm{y}$, la corrección de primer orden está dada por (Huang \& Haus, 1990).

$$
\beta_{i}-\breve{\beta}_{i}=\frac{\int \frac{1}{n_{x}^{2}} \frac{\partial n_{i}^{2}}{\partial x_{i}} \frac{\partial \widetilde{E}_{i}^{*}}{\partial x_{i}} \widetilde{E}_{i} d x d y}{\int\left|\widetilde{E}_{i}\right|^{2} d x d y}
$$

donde $i$ y $x_{i}$ son igual a x o y para modos polarizados en $x$ o $y$, respectivamente.

El parámetro de dispersión $D$ de una guía de onda se puede calcular a partir de $n_{\text {eff }}=\beta_{i} / k_{0}$ en función de la longitud de onda a través de la siguiente expresión:

$$
D=-\frac{\lambda}{c} \frac{d^{2} n_{e f f}}{d \lambda^{2}}
$$

donde c es la velocidad de la luz en el vacío. Para incluir el efecto de la dispersión cromática del material en la ecuación (6), se utilizó la ecuación de Sellmeier con los coeficientes reportados en la literatura para el silicio (Agrawal, 1995).

\section{Resultados}

Para probar nuestro método, se analizó una fibra óptica microestructurada multimodo que consiste en tres huecos anulares alrededor de un núcleo sólido de silicio (ver Figura 8(a)). Los huecos tienen un radio interior de $1 \mu \mathrm{m}$, un radio exterior de $2 \mu \mathrm{m}$ y un 
ancho angular de $108^{\circ}$ (Figura $8(\mathrm{~b})$ ). La simetría que presenta esta estructura permite que la mitad de la estructura pueda ser considerada, finalmente se usó como dominio computacional un semicírculo de $2.05 \mu \mathrm{m}$ de radio (Figura 8(c)).

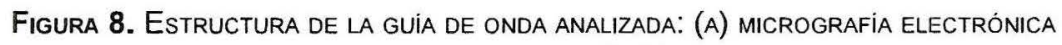
TOMADA DE LA REFERENCIA (LIGHT READING); (B) MODELO COMPUTACIONAL;

(c) DISCRETIZACIÓN DEL MODELO COMPUTACIONAL UTILIZADO

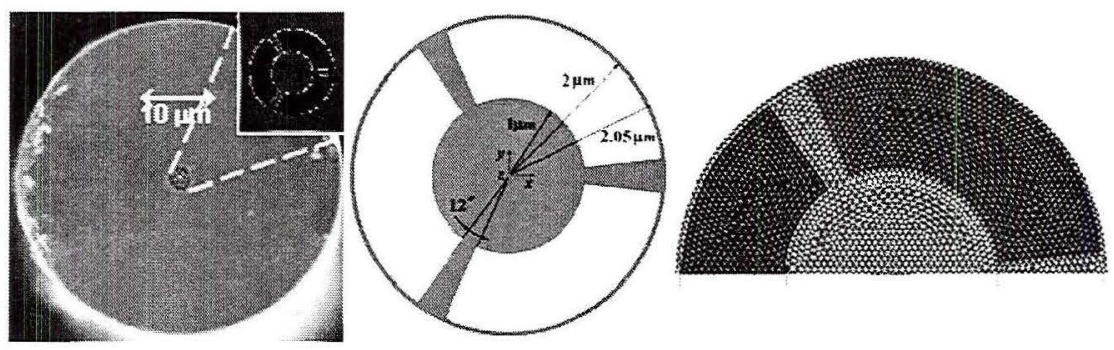

En la Figura 9 se comparan los resultados obtenidos del parámetro de dispersión de los modos tipo $\mathrm{HE}_{11}, \mathrm{TE}_{01} \mathrm{y} \mathrm{HE}_{21}$, con los reportados en la referencia (Uranus \& Hoekstr, 2004), sobre un rango de longitud de onda desde $\lambda=0.4 \mu \mathrm{m}$ a $\lambda=1.6 \mu \mathrm{m}$. Nótese que esta estructura tiene una alta fracción de llenado de aire, por lo que el acople entre las componentes ortogonales del campo eléctrico es fuerte, y por ende, el análisis escalar es inapropiado en el rango de análisis de la longitud de onda. Este hecho se observa más claramente en las curvas de dispersión para los modos excitados $\mathrm{TE}_{01} \mathrm{y} \mathrm{HE}_{21}$. Por el contrario, la solución escalar corregida concuerda muy bien con la solución vectorial, excepto para el modo menos confinado, el $\mathrm{HE}_{21}$, y para $\mathrm{TE}_{01}$ comienza a haber divergencia a partir de $\lambda>1.45 \mu \mathrm{m}$. Este hecho se debe a que el campo de estos modos se extiende más allá de los huecos de aire, tal como se muestra en las Figuras 10, 11 y 12, que hace que los efectos por polarización se incrementan fuertemente. Es claro que, en estas condiciones la validez de nuestra propuesta se reduce, ya que la corrección propuesta no es suficiente para dar cuenta de los efectos 
por polarización; sin embargo, los resultados obtenidos no difieren significativamente de la solución vectorial.

Figura 9. PARÁmETRO DE DISPERSIÓN, PARA LA ESTRUCTURA ANALIZADA, DE LOS MODOS TIPO $\mathrm{HE}_{11}, \mathrm{TE}_{01} Y \mathrm{HE}_{21}$

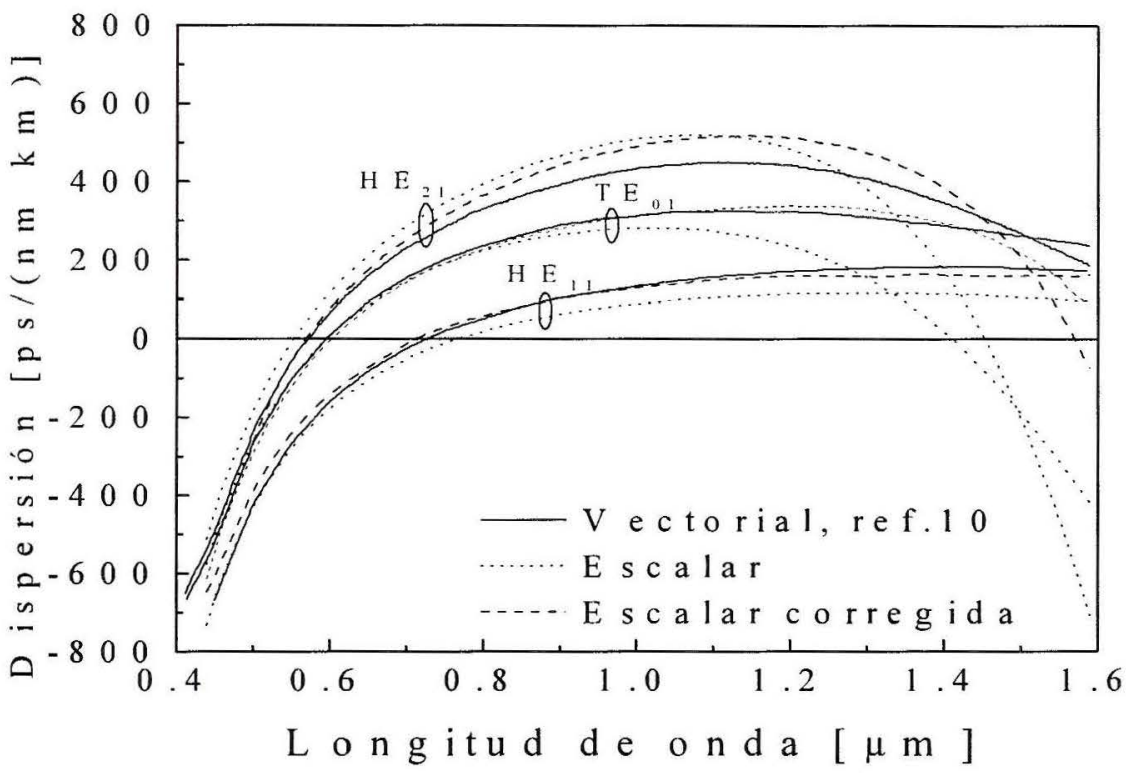

Figura 10. Degradado del PERFIL DE INTENSIDAD DEL CAMPO ElÉCTRICO NORMALIZAdo, CALCULADO CON LA METODOLOGIA PROPUESTA PARA EL MODO TIPO HE ${ }_{11}$, PARA LAS LONGITUDES DE ONDA (a) $\lambda=0.6 \mu \mathrm{m}$, (b) $\lambda=1.0 \mu \mathrm{m}$, (c) $\lambda=1.6 \mu \mathrm{m}$

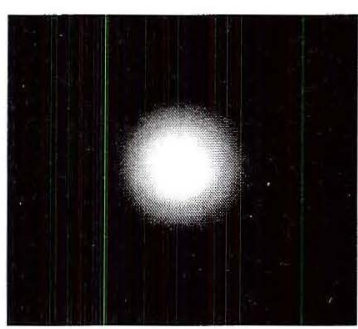

(a)

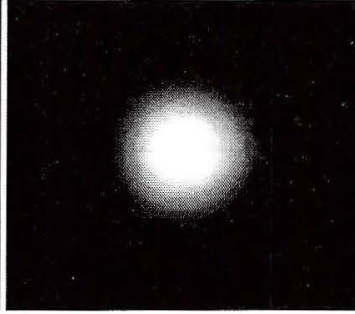

(b)

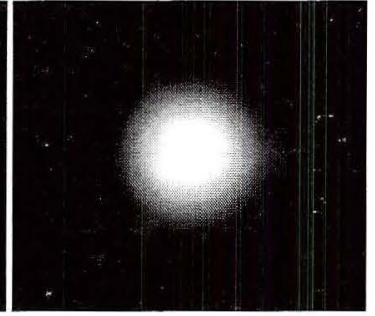

(c) 
Figura 11. Degradado del Perfil de INTENSIDAD del CAMPo ElÉctrico Normalizado, CALCULADO CON LA METODOLOGIA PROPUESTA PARA EL MODO TIPO TE ${ }_{01}$, PARA LAS LONGITUDES DE ONDA (a) $\Lambda=0.6 \mu \mathrm{m}$, (b) $\lambda=1.0 \mu \mathrm{m}$, (c) $\lambda=1.6 \mu \mathrm{m}$

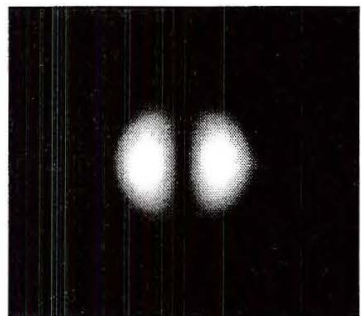

(a)

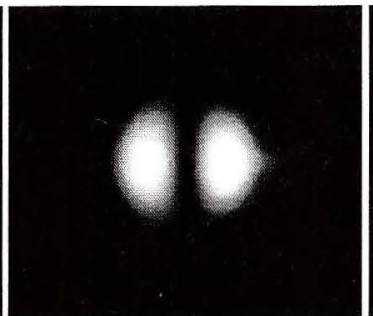

(b)

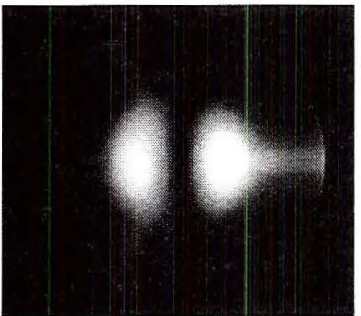

(c)

Figura 12. Degradado del PERFIL de INTENSIDAD del CAMPo ElÉctrico NORMALIZADO, CALCULADO CON LA METODOLOGIA PROPUESTA PARA EL MODO TIPO HE ${ }_{21}$, PARA LAS LONGITUDES DE ONDA (A) $\lambda=0.6 \mu \mathrm{m}$, (b) $\lambda=1.0 \mu \mathrm{m}$, (c) $\lambda=1.6 \mu \mathrm{m}$

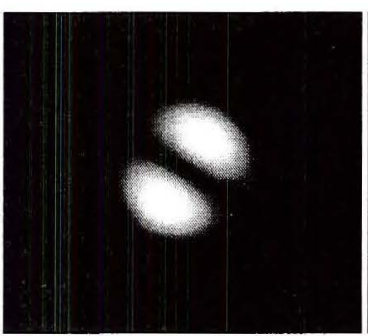

(a)

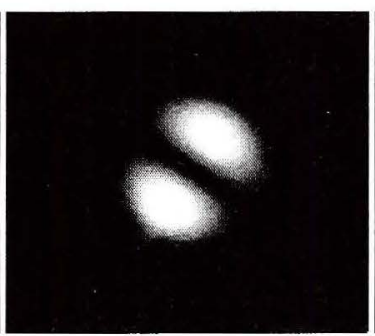

(b)

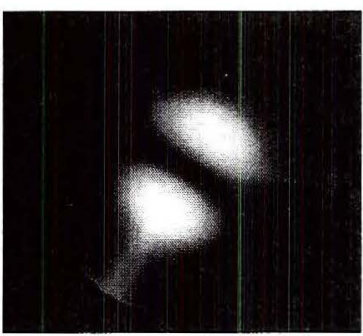

(c)

\section{CONCLUSIONES}

Con el avance tecnológico en la fabricación de guías de ondas ópticas, se abren nuevos retos debido a que estos nuevos sistemas presentan comportamientos ópticos inusuales y ventajas jamás imaginadas, que no son posibles lograrlos con las fibras ópticas convencionales, situación que permite novedosas soluciones a aplicaciones en telecomunicaciones, tales como el control de la dispersión en los pulsos de luz, disminución de las pérdidas en el medio de transmisión, la operación en el régimen mono-modo, 
nuevas fuentes de luz completamente integrada a fibra óptica, etcétera. En el área de la sensórica, los dispositivos ópticos cada vez, van ganando más campo de acción debido a que son sistemas muy sensibles a parámetros de interés y de fácil control, además de existir aplicaciones donde es imposible llegar si no es con esta tecnología; por ejemplo, el monitoreo de combustibles y sustancias en medios altamente corrosivos y peligrosos.

Todo esto ha sido propiciado por ideas novedosas, acompañadas de un estudio que se ha llevado a cabo por medio de modelos computacionales con el fin de seguir diseñando y optimizando nuevas fibras ópticas especiales, para futuras aplicaciones. De acuerdo con el propósito de este trabajo, se ha aplicado una metodología basada en el método de elementos finitos escalar que incluye una corrección a la constante de propagación, que da cuenta de los efectos de polarización para analizar cualquier tipo de guía de onda dieléctrica, aun, las micro-estructuradas. Los resultados obtenidos para el parámetro de dispersión de una guía onda óptica multimodo que consiste de tres huecos anulares alrededor de un núcleo sólido son aceptables. Esta herramienta computacional se puede utilizar para investigar las propiedades de propagación en estas guías de onda especiales; en particular, se puede explorar la posibilidad de diseñar guías de onda de área efectiva grande y que sigan siendo monomodo, o con área efectiva muy pequeña, para aumentar la no linealidad óptica, también guías que presenten alta birrefringencia, fibras que presenten altos valores de dispersión negativa con miras a emplearse como compensadores de dispersión en los sistemas de comunicaciones, y así un gran número de aplicaciones.

\section{Agradecimientos}

Al Dr. Pedro Ignacio Torres Trujillo, por su acompañamiento en este trabajo, y a la Universidad Nacional de Colombia por su apoyo a través del programa de Becas Nacionales, para estudiantes sobresalientes de posgrado. 


\section{Tramanos CITADOS}

Agrawal, G. P. (1995). Nonlinear Fiber Optics (2nd ed.). New York: Academic.

Alivizatos, E. G., Chremmos, I. D., Tsitsas, N. L., \& Uzunoglu, N. K. (2004). Green's-function method for the analysis of propagation in holey fibers. Optical Society of America A., 21 (5), 847-857.

Argyros, A., Bassett, I. M., Van Eijkelenborg, M. A., \& Large M, C. J. (2004). Analysis of ring - structures Bragg fibres for single TE mode guidance. Optics Express, 12, 2688-2698.

Aristizábal, V. H., Vélez, F. J., \& Torres, P. I. (2006). Analysis of photonic crystal fibers: Scalar solution and polarization correction. Optics Express, 14, 11848-11854.

Aristizábal, V. H., Velez, F. J., \& Torres, P. I. (2004). Modelling of photonic crystal fibers with the scalar finite element method, in $5^{\text {th }}$ Iberoamerican Meeting on Optics and $8^{\text {th }}$ Latin American Meeting on Optics, Laser an their Applications. A. Marcano and J.L. Paz, eds., Proc. SPIE 5622.

Birks, T. A., Bird, D. M., Hedley, T. D., Pottage, J. M., \& Russell, P. (2004). Scaling laws and vector effects in bandgap-guiding fibres. Optics Express, $12,69-74$.

Birks, T. A., Knight, J. C., \& Russell, P. (1997). Endlessly single-mode photonic crystal fiber. Optics letters, 22, 961-963.

Brechet, F., Marcou, J., Pagnoux, D., \& Roy, P. (2000). Complete analysis of the characteristics of propagation into photonics crystal fibers. Optical Fiber Technology, 6, 181-183.

Campbell, S., McPhedran, R. C., De Sterke, C. M., \& Botten, L. C. (2004). Differential multipole method for microstructured optical fibers. Journal of the Optical Society of America B, 21 (11), 1919-1928.

Corporation Penn Well. (August de 2004). Photonic-crystal fibers have many uses. Optoelectronics World Magazine.

Cregan, R. F., Manga, B. J., Knight, J. C., Birks, T. A., Russell, P., Roberts, P. J., y otros. (1999). Single-mode photonic band gap guiden of light in air. Science, 285, 1537-1539.

Crystal Fibre. (2008). Recuperado el 17 de February de 2008, de Tutorial - high index guiding fibers: http://www.crystal-fibre.com/technology/_tutorial2.shtm 
Crystal Fibre. (2008). Recuperado el 17 de February de 2008, de Crystal fibre: http://www.cystal-fibre.com

Crystal Fibre. (2008). Recuperado el 17 de February de 2008, de Fabrication: http://www.crystal-fibre.com/support/faq.shtm

Cucinotta, A., Selleri, S., Vincetti, L., \& Zoboli, M. (2002). Holey fiber analysis through the finite-element method. IEEE Photonics Technology Letters, 14, 1530-1532.

Derickson, D. (1998). Fiber optics, test and measurements (1 ed.). New Yersey: Prentice Hall.

Fujisawa, T., \& Koshiba, M. (2003). Finite element characterization of chromatic dispersion in nonlinear holey fibers. Optics Express, 11, 14811489.

Gander, M. J., McBride, R., Jones, J. D., Mogilevtsev, D., Birks, T. A., Knight, J. C., y otros. (1999). Experimental measurement of group velocity dispersion in photonic crystal fiber. Electronics Letters, 35, 63-64.

Gavea Sensors. (2008). Recuperado el 17 de February de 2008, de Aplicaciones: http://www.gaveasensors.com.br/aplicacoes.html

Huang, W., \& Haus, H. (1990). Analysis of buried-channel waveguides and couplers: scalar solutionand polarization correction. Journal of Lightwave Technology, 8, 642-648.

Huttunen, A., \& Torma, P. (2005). Optimization of dual-core and microstructure fiber geometries for dispersion compensation and large mode area. Optics Express, 13, 627-635.

Kerbage, C. E., Eggleton, B. J., Westbrook, P. S., \& Windeler, R. S. (2000). Experimental and scalar beam propagation analysis of an airsilica microstructure fiber. Optics Express, 7, 113-122.

Kinight, J. C., Arriaga, J., Birks, T. A., Ortigosa-Blanch, A., Wadsworth, W. J., \& Russell, P. (2000). Anomalous dispersion in photonic crystal fiber. IEEE Photonics Technology Letters, 12, 807-89.

Knight, J. C., Birks, T. A., Russell, P., \& Atkin, D. M. (1996). All-silica single-mode optical fiber with photonic crystal cladding. Optics letters, 21, 1547-1549.

Knight, J. C., Birks, T. A., Russell, P., \& Atkin, D. M. (1996). Pure silica single-mode fibre with hexagonal photonic crystal cladding. Optics Letters, 21, (1547-1579).

Knight, J. C., Broeng, J., Birks, T. A., \& Russell, P. (1998). Photonic band gap guidance in optical fiber. Science, 282, 1476-1478. 
Koshiba, M., \& Tsuji, Y. (2000). Curvilinear hybrid edge/nodal elements with triangular shape for guided-wave problemes. Journal of Lightwave Technology, 18, 737-743.

Laser Focus World. (2008). Recuperado el 17 de February de 2008, de Microstructure fibers: photonic-crystal fibers move out of the laboratory: http://www.laserfocusworld.com/articles/289408.

Ligth Reading. (2008). Recuperado el 17 de February de 2008, de Speciality fiber: http://www.lightreading.com/topics.asp?node_id=1057

Mogilevtsev, D., Birks, T. A., \& Russell, P. (1999). Localized function method for modeling defect modes in 2-D photonic crystals. Journal of Lightwave Technology, 17, 2078-2081.

Monro, T. M., Richardson, D. J., Broderick, N. G., \& Bennett, P. J. (1999). Holey optical fibers: An efficient modal model. Journal of Lightwave Technology, 17, 1093-1102.

Monro, T. M., Richardson, D. J., Broderick, N. G., \& Bennett, P. J. (2000). Modeling large air fraction holey optical fibers. Journal of Lightwave Technology, 18, 50-56.

Network Cabling Help. (2008). Recuperado el 17 de February de 2008, de Fibre optics: http://www.datacottage.com/nch/fibre.htm

NGA and NASA. (2006). Obtenido de The Shuttle Radar Topography Mission (SRTM). Project Formulation \& Management Applications (PFMA) group: http://www2.jpl.nasa.gov/srtm

Ortigosa, A., Diez, A., Delgado, M., Cruz, J. L., \& Andres, M. V. (2004). Nonlinear microstructured fibers with ultrahigh birefrigence. Proc. SPIE , $5622,855-858$.

Precision Technologies. (2008). Recuperado el 17 de February de 2008, de Newport corporation lauches PCFs product line: http://uk.precisionnews. com/article_Trackballs-industriels-CKS_274.html

Rahman, B. M., \& Davies, J. B. (1984). Penalty function improvement of waveguide solution by finite elements. IEEE Transactions on Microwave theory and Techniques, 32, 922-929.

Reeves, W., Knight, J. C., Russell, P., \& Roberts, P. J. (2002). Demonstration of ultra-flattened dispersion in photonic crystal fibers. Optics Express, 10, 609-613.

Riishede, J., Mortensen, N. A., \& Laegsgaard, J. N. (2003). A 'poor man's approach' to modelling microstructured optical fibres. Journal of Optics A: Pure and Applied Optics, 5, 534-538. 
Russell, P. (2003). Photonic Crystal Fibers. Science, 299, 358-362.

Saitoh, K., \& Koshiba, M. (2002). Full-vectorial imaginary-distance beam propagating method based on a finite element scheme:applications to photonics crystal fibers. IEEE Journal of Quantum Electronics, 38, 927933.

Saitoh, K., \& Koshiba, M. (2003). Single-polarization single-mode photonic crystal fibers. IEEE Photonics Tecnhnology Letters, 15, 1384-1386.

Saitoh, K., Koshiba, M., Hasegawa, T., \& Sasaoka, E. (2003). Chromatic dispersion control in photonic crystal fiber: application to ultra-flattened dispersion. Optics Express, 11, 843-852.

Schott. (2008). We make things visible. Recuperado el 17 de February de 2008, de http://www.us.schott.com/fiberoptics/english/index.html?c=aL.

Silvestre, E., Andres, M. V., \& Andres, P. (1998). Biothonormal-basis method for the vector description of optical -fiber modes. Journal of Lightwave Technology, 16, 923-928.

Steel, M. J., White, T. P., De Sterke, C. M., McPhedran, R. C., \& Botten, L. C. (2001). Symmetry and degeneracy in microstructured optical fibers. Optics Letters, 26, 488-490.

Thomasnet. (2008). Recuperado el 17 de February de 2008, de Photonic Crystal Fibers support high gain fiber lasers: http://news.thomasnet. com/fullstory/453098

Uranus, H. P., \& Hoekstra, H. J. (2004). Modelling of microstructured waveguides using a finite-element-based vectorial mode solver with transparent boudary conditions. Optics Express, 12, 2795-2809.

Yio. (2008). Fibras ópticas. Recuperado el 17 de Febrero de 2008, de http:// www.yio.com.ar/fibras-opticas/index.php

Yu, C. P., \& Chang, H. C. (2004). Applications of the finite difference mode solution method to photonic crystal structures. Opt. Quantum Electron, $36,145-163$. 
